\title{
LA INJUSTICIA Y LA POLÍTICA DE LA DIFERENCIA*
}

\author{
CARLOS ALBERTO MOLINARO**
}

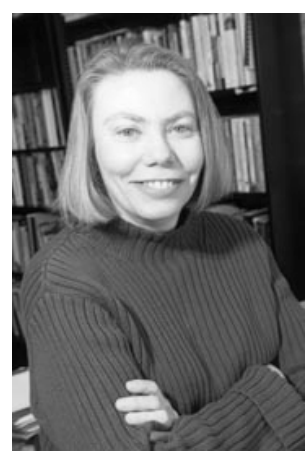

Iris Marion Young (1949 - 2006), que tuvimos el privilegio de conocer personalmente, fue una filósofa y científica-política estadunidense de gran sensibilidad intelectual. Era profesora de Ciencias Políticas de la Universidad de Chicago y afiliada al Gender Studies Center (Centro de Estudios de Género) e al Programa de Derechos Humanos. Su investigación abarcaba la Teoría Política Contemporánea, Feminismo y análisis normativo de Políticas Públicas. Falleció en 2006, después de una lucha de más de año contra un cáncer de esófago.

Este es un gran libro. En él percibimos que el debate entre feminismo y multiculturalismo ha estado especialmente vivo en los últimos años y ha apuntado dos direcciones en conflicto: de un lado, el discurso del multiculturalismo y, de otro, la exigencia de reconocimiento de la diferencia cultural, sobre todo cuando está asociada a desigualdades e injusticias. De hecho, la obra de Iris Marion Young Justice and the Politics of Difference, publicada en Estados Unidos en 1990, y traducida al castellano diez años después, representó la necesidad de ampliar el marco problemático feminista para incorporar el cruce entre género y cultura, entre otros, como suma de vectores de opresión. Esta obra continua actualísima y debe ser leída por todos aquellos que se interesan por el tema de la justica y la política de la diferencia.

Es un libro de gran densidad, consta de ocho capítulos e un epílogo sobre la Justicia Internacional. El compromiso de Young es demonstrar la amplitud del significado de justicia, pues en su entendimiento las teorías filosóficas contemporáneas se encuentran limitadas en el análisis de la justicia en su dimensión distributiva que no alcanza el actual sentido de justicia social que implica la "eliminación de la dominación y la opresión institucionalizada”. Al hacer el análisis del modelo distributivo “por 'paradigma' entiende una configuración de elementos y prácticas

* Breve reseña para impartir en las clases del Doctorado en Derechos Humanos y Desarrollo, UPO-ES, en el mes de enero/febrero de 2007.

** Doctor en Derecho. Profesor de la PUCRS. Profesor del Programa de Doctorado en Derechos Humanos y Desarrollo de la UPO-ES 
que definen una investigación: presuposiciones metafísicas, terminología no cuestionada, preguntas características, líneas de razonamiento, teorías específicas y su ámbito y modo de aplicación característica". En ese enfoque afirma que el centro de análisis de la cuestión de la justicia es la distribución de los bienes y encargos sociales según parámetros moralmente aceptados, ocupando los bienes materiales posición de destaque. Examinando las teorías de justicia observa Young la primacía central de la perspectiva de distributividad. Así, en Rawls, Runcimann, Ackerman y William Galston, siendo en ese último, se encuentra de modo explícito la relación entre justicia y distribución. Según Galston, la justicia incluí “un conjunto de relaciones de posesión” - en una de esas relaciones el individuo es distinto del objeto poseído, consecuentemente, para Galston la justicia es, "la adecuada asignación de entidades a los individuos; la calidad de adecuadas se refiere tanto a la relación entre algunos aspectos de las entidades y los individuos en cuestión como la relación entre dichas entidades y los posibles modos de asignación. El conjunto de las entidades podría incluir objetos, cualidades, posiciones dentro del sistema o incluso seres humanos". ${ }^{1}$ A seguir Young anota que la identidad conceptual de justicia y distribución es observada inclusive entre los liberales y hasta mismo, bajo una óptica socialista de bies marxista. Con relación a Walzer, ${ }^{2}$ afirma que su posición es ambigua cuando acepta la correlación justicia/distribución, asegura que los bienes dominantes deberían ser mejor distribuidos, es decir, el monopolio es injusto. El paradigma distributivo no distingue los individuos y/o las situaciones. El modelo es siempre lo mismo. Esos hechos llevan al establecimiento de una relación entre los individuos y los bienes, bien como la cuantidad de bienes poseídos. Para Young, el paradigma distributivo presenta dos problemas fundamentales: "primero, dicho paradigma tiende a ignorar el contexto institucional que determina la distribución material, al mismo tiempo que con frecuencia lo presupone; secundo, cuando el paradigma se aplica a bienes y recursos no materiales la lógica de la distribución los tergiversa”. Tenemos, pues, que el aspecto de la distribución de bienes no puede ser totalmente apartado del concepto de justicia. La gran mayoría de las teorías respecto de la justicia social tienen por punto central la distribución de recursos materiales y de las posiciones de destaque. En un mundo en que parcelas considerables de la populación están muriendo de hambre no es posible hablar de justicia social sin una distribución de bienes que asegure la supervivencia. Todavía, en sociedades más avanzadas donde las pretensiones de los grupos sociales son de naturaleza diversa, no se busca más la distribución de bienes materiales. Las reivindicaciones son de otra orden, comprenden las cuestiones de poder, de tomada de decisiones, de imagen, de división y significado del trabajo. Esos aspectos no están comprendidos cuando se establece la identidad justicia/distribución, lo que torna impropio el modelo distributivo. Las criticas al paradigma distributivo son tanto con relación a la incapacidad de valuar las

\footnotetext{
${ }^{1}$ Galston ha intentado desarrollar una modalidad de pensamiento liberal fuertemente abierto al comunitarismo. En general, su intento ha despertado más insatisfacciones que aprobación: para los críticos del liberalismo, ella parece insuficientemente comunitarista, mientras que a ojos de los liberales resulta excesivamente concesiva (a propósito consulte-se su libro: Liberal Purposes. Goods, Virtues, and Diversity in the Liberal State. Cambridge: Cambridge University Press. 1991; más recentemente: Liberal Pluralism. The Implications of Value Pluralism for Theory and Practice. Cambridge: Cambridge University Press, 2002).

${ }^{2}$ Cf. Walzer, Michael: Spheres of Justice. New York, Basic Books, 1983
} 
instituciones capitalistas y las relaciones de clase (v. g. Allen Wood ${ }^{3}$ ) como por el hecho de ocultaren las desigualdades que no son capaces de valuar (Macpherson ${ }^{4}$ ). La crítica del modelo distributivo bajo la óptica marxista sustenta que la justicia es un concepto de la ideología burguesa y consecuentemente irrelevante para un análisis socialista. Lo que interesa para la autora, todavía, es el hecho que aún que diversa la ideología no ha discusión, cuando se trata de establecer el significado de justicia, sino la aceptación de las relaciones de producción que definen el modelo económico. El análisis marxista del paradigma distributivo es tímido. La ausencia de las relaciones capitalistas de clase no es la única en el sentido distributivo, otras se hacen sentir como las familiares y las resultantes del trabajo. El contexto social a ser considerado debe ser amplio, bien como el institucional no puede estar limitado al sistema de producción. Deben, igualmente, ser consideradas las cuestiones relativas a la tomada de decisiones y la división del trabajo. Esos aspectos que extrapolan la distribución de bienes pueden y deben ser considerados por las teorías distributivas, lo que es realizado por muchos teóricos, a ejemplo de Rawls, David Muller y Galston. Esta consideración, todavía, en el ámbito del paradigma distributivo no se aparte de la noción de distribuir y considera los bienes inmateriales como materiales, centrándose la cuestión en la distribución entre los agentes, pues según Young, lleva a una “ontología social equivocada o incompleta”. Lo sistema distributivo trae en si el pretexto de que los juicios sociales son juicios de valor que tienen el individuo en cuanto comparado con otro lo que aparta la consideración y la reflexión respecto de lo que hace este mismo individuo.

La consideración de los bienes materiales en el paradigma distributivo es hecha, en la mayoría de las veces, considerando los derechos, las oportunidades y la autoestima. Cuanto a los derechos la impropiedad radica en que su inclusión no se puede dar como se fuera una "cosa”, ya que la autora, entiende que son relaciones, reglas institucionalizadas que disciplinan el comportamiento de unos con relación a los otros. Cuanto a las oportunidades no se encuentra bien definida la cuestión. Considerando la oportunidad como “ocasión” es posible decir, que algunos tienen más que otros y así, mejor pueden adaptarse al paradigma distributivo. Se, todavía, tuviere oportunidad como capacidad no se estará dando viabilidad a la idea distributiva, pues la oportunidad está relacionada con las normas que disciplinan la acción y la forma como se posiciona y es considerado el individuo en el contexto social. De igual forma, la autoestima es un bien inmensurable lo que aparta la idea distributiva. Por otro lado, no se puede disociar la autoestima de la propia persona para atribuirle como se un bien fuese. La autoestima es una actitud de la propia persona con relación a si misma y la vida. La consideración de los derechos, de las oportunidades y de la autoestima como pasibles de distribución, según Young, encubre las bases sociales e institucionales de esos valores. Aún - dice la autora - los críticos mencionarían que no se tratan de bienes y sino de poder social, sin embargo, no se encuentran los

${ }^{3}$ Cf. Wood, Allen, Marx and Equality, in, John Mepham and David Hillel-Ruben (eds.), Issues in Marxist Philosophy v. 4. Sussex: Harvester Press, 1981.

${ }^{4}$ Cf. Macpherson, Crawford Brough. The Rise and Fall of Economic Justice. Oxford: Oxford University Press, 1985

DIREITOS FUnDAMENTAIS E JUSTIÇA N 2 - JAN./MAR. 2008 
teóricos en consonancia con la manera de enfocar el poder. Algunos lo excluyen cuando conceptúan la justicia, otros lo incluyen. Señala la autora, que el poder -en la división distributiva- considerado como bien aparta la cuestión de la dominación y permite que se concentre en manos de pocas personas. Apartar la injusticia seria redistribuir el poder y no apartar la opresión y la dominación.

Con la premisa de que opresión y dominación son contenidos de la injusticia y son conceptos que se sobreponen -aún que posan ser distinguidos- la autora en su capítulo quinto, sobre el título, "Las cinco caras de la opresión”, hace el análisis de la explotación, marginación, carencia de poder, imperialismo cultural y violencia, examina en preliminar, el significado y el contenido de la expresión opresión como utilizada por los movimientos sociales de los EE.UU. en los anos 60's. Los movimientos son: mujeres, gente negra, chicanos, latinos, indígenas, judíos, lesbianas, gays, gente árabe, asiáticos, mayores, gente de clase obrera, discapacitados físicos y mentales, etc., sobre esos movimientos, la autora, objetiva identificar la sistematización del concepto de opresión tal como utilizado por los diversos movimientos políticos, proporcionando los argumentos normativos para la aclaración de los usos inadecuados de las expresiones. Así, la opresión está directamente vinculada a características de los grupos sociales como la utilizan. Por tanto, para la definición del término opresión hace necesario definir los grupos sociales. Lo hace Young, definiendo previamente los conjuntos y las asociaciones. Los grupos no son meros conjuntos. No se reúnen, por ejemplo, los negros americanos solamente en razón de un atributo común, el color de la piel que los hace un simple conjunto, existe algo más, existe la afinidad. De igual forma, los grupos no son una asociación, la asociación presupone la existencia del individuo ya formada que se integra a la organización. El grupo precede al individuo. Los grupos no son únicos, son múltiplos. Comportan divisiones en su propio interior, principalmente en las grandes y complejas sociedades. Algunos se forman en razón de la opresión, más su existencia no es por si solo, factor de opresión. Puesta la discusión respecto del concepto de grupo social, pasa Young al examen de "las caras de la opresión". Sobre la explotación, afirma que la sociedad capitalista actual aparta la existencia de clases institucionalizadas como ocurría en las que la antecedieran. Tal hecho, todavía, no aparta la existencia de diferencias entre los individuos. Hay personas ricas que son los propietarios de los medios de producción y un gran número de individuos que trabajan, que generan esa producción. Esta división entre los propietarios y los que producen, en una sociedad en que inexisten clases sociales de forma institucionalizada es el objeto de la teoría de la explotación desarrollada por Marx. Los bienes se cambian por su valor. Esta particularidad, cambio por el valor, torna enigmática la existencia del lucro, base del sistema capitalista. La solución del enigma es encontrada en la teoría del valor de la fuerza del trabajo. El bien no vale la fuerza del trabajo que se agota en la producción, el bien vale en razón del número de horas de trabajo. Ocurre, con todo, que el quantum pago por la hora de trabajo no es el equivalente al valor del bien, y esta diferencia -el valor excedente- que es apropiado por el propietario del capital, que caracteriza el lucro, es la base del sistema capitalista. Sin embargo, hasta hoy, concurren controversias entre los teóricos marxistas con respecto a la teoría de la explotación fundamentada en el 
trabajo, por ejemplo, Macpherson reconstruye la teoría dándole un carácter normativo. Según Young, la injusticia está en la sumisión y en la transferencia de poder. La justicia, afirma la autora, requiere "la eliminación de las formas institucionales que permiten y refuerzan ese proceso de transferencia y su reemplazo por formas institucionales que permitan a todas las personas desarrollar y usar sus capacidades en un modo que no prive a otras personas sino que les permita desarrollo y usos similares". De modo que la opresión proviene de un proceso de transferencia de los resultados del trabajo de un grupo a favor de otro. La teoría marxista, centrada en el trabajo, no contempla como lo exponen algunos teóricos, a totalidad de las formas de opresión. Hay opresión, sexual, racial, que en principio no se encuentran incluidas en la idea de transferencia de la forma de trabajo, lo que muchas veces es apartado por las feministas. Entienden esas, que las mujeres viven en constante cambio y transferencia no solo con relación a los frutos de sus trabajos, como también con relación a las energías sexuales y de educación. La autora cita Christine Delphy que define matrimonio como: "una relación de clase en la que el trabajo de la mujer beneficia al hombre sin que haya una remuneración comparable". ${ }^{5}$ Respecto a la explotación de género la casi totalidad de las teorías toma por punto de partida las relaciones familiares. Hoy, ya se apartan las teorías del ámbito familiar y se examina la explotación de género en las relaciones con el Estado y en las relaciones de trabajo. Al lado de la opresión de género y de aquella de clase hay la opresión en razón de raza. La opresión de raza, ha se exteriorizado principalmente a través del trabajo no solo por la distinción de remuneración, sino, principalmente, por la tarea destinada. La opresión tiene sido demostrada en las sociedades racistas, principalmente, por la destinación de los grupos discriminados de las tareas de servir. Al fin y al cabo, Young, dice: "hacer justicia donde hay explotación requiere reorganizar las instituciones y las practicas de toma de decisiones, modificar la división del trabajo, y tomar medidas similares para el cambio institucional, estructural y cultural".

Otra forma, es la marginación. Young entiende marginación como la opresión a que son sometidos aquellos cuyo el mercado de trabajo, o no puede, o no quiere emplear. Actualmente, en el ámbito estadounidense la opresión racial se exterioriza mucho más por la marginación do que por la explotación. No son, sin embargo, solamente los integrantes de determinados grupos raciales los sometidos a la marginación. Es elevado el número de mayores, jóvenes, madres solteras, minusválidos que no obteniendo trabajo se encuentran apartados del proceso de producción, victimas de la opresión. La marginación lleva a la privación de bienes materiales, crea la dependencia, lo que, sin duda, afecta la propia ciudadanía. La dependencia, todavía, no es por esencia opresiva, hay situaciones en que ella se torna imprescindible, no se caracterizando como opresión (caso de enfermos, de niños pequeños, etc.). La dependencia no generadora de opresión fue examinada por las teorías feministas cuyo modelo, segundo Held, citado por la autora, “concibe la justicia como el reconocimiento de respecto y participación en la toma de decisiones tanto quiénes

\footnotetext{
${ }^{5}$ A propósito consúltese el excelente artículo de Christine Delphy, Penser le genre: quels problèmes, in: Sexe et genre, De la hiérarchie entre les sexes. Direction de Marie-Claude Hurtig, Michèle Kail et Hélène Rouch. Paris: Éditions du CNRS, 1991.
} 
son dependientes como a quiénes son independientes”. Aún que no ocurra marginación cuando hay dependencia, no se puede afirmar que no exista marginación siempre que haya la satisfacción de las necesidades básicas, eso porque permanece la no-participación en la sociedad. La marginación viene se acentuando haciendo con que algunos analistas de política social propongan desvincularla del sistema general de salarios con la creación de un salario social para contemplar aquellos que están fuera del mercado de trabajo.

Ahora, otra cosa es la carencia de poder. La división del trabajo sea ella considerada como proveniente de la apropiación del trabajo de uno por otros, sea la existente en la sociedad contemporánea que se refleja en clases sociales -afirma Youngorigina una de las caras de la opresión: carencia de poder. En la sociedad contemporánea la división de trabajo entre profesionales y no-profesionales condiciona un grande número de trabajadores a una ausencia de participación en las decisiones que influencian directamente sus vidas. Carentes de poder reciben continuamente ordenes y eventualmente las dan. No disponen de autonomía en el trabajo, no tienen espacio para la creatividad. La carencia de poder como elemento caracterizador de opresión, fue examinada por Sennett y Cobb ${ }^{6}$, en un profundo estudio sobre los hombres de clase obrera. Los carentes de poder no poseen autoridad, status y sentido de si mismo como poseen los profesionales. La profesionalización presupone en la mayoría de las veces una educación universitaria. Aún que en muchas hipótesis el profesional no disponga de autonomía, generalmente, el trabajo en nivel profesional implica una parcela de poder. Además, el ejercicio del poder en la sociedad moderna, la división del trabajo en mental y manual fundamenta también la división en clases media y obrera, lo que extrapola en mucho la división del trabajo, pudiendo hasta determinar el local en que será su vivienda, se profesional u obrera. La profesionalización agrega también, según la autora, la respetabilidad que es toda una forma de vida. En lo cotidiano las mujeres y las personas de color deben probar su respetabilidad, lo que ya se encuentra presumido tratándose de hombre blanco y profesional.

Otra característica, afirma Young, es el imperialismo cultural. Los movimientos sociales de liberación, especialmente los feministas y aquellos de negros, tienen identificados otras formas de opresión que se manifiesta por la universalización de la experiencia y cultura del grupo dominante imposta como norma. A ese hecho denomina Young de imperialismo cultural. La sociedad pasa a moverse y a reflexionar las experiencias no de todos, sino del grupo dominante. La existencia de otros grupos puede afectar aquél dominante el cual reaccionando por la imposición de su modelo toma las diferencias de cada grupo como desvío o inferioridad, pasando a designar aquellos por "los otros”. La dominación por medio de la cultura lleva a la formación de estéreotipos. Los individuos son apriorísticamente clasificados según un estereotipo formado. El propio integrante del grupo se ve según los padrones del grupo dominante. A esta experiencia du Bois ${ }^{7}$, citado por la autora, llamó de "doble conciencia". Lo dominado es reconocido por su cultura. Ahí, existe con una doble conciencia

${ }^{6}$ Cf. Sennett, Richard; Cobb, Jonathan. The Hidden Injuries of Class. New York: Vintage Books, 1972

${ }^{7}$ Cf. Du Bois, William Edward Burghardt. The Souls of Black Folk. Chicago: A.C. McClurg \& Co. [Cambridge]: University Press John Wilson and Son, Cambridge, U.S.A., 1903. 
porque sabe de la existencia de su grupo, de sus hábitos, de su cultura al mismo tiempo que tiene conciencia que hay un grupo diverso, cuyos padrones son los dominantes y estos establecieron los padrones de estereotipos. Resulta aunque del imperialismo cultural la invisibilidad del dominado, pues el grupo dominante no reconoce el dominado como identidad cultural. No existe en lo que toca a los oprimidos, experiencias que afecten el grupo dominante, generándose así las injusticias de la imposición del padrón cultural.

La violencia es otra característica. Las teorías respecto de la justicia no mencionan la violencia a que están sometidos los integrantes de determinados grupos por el simple hecho de pertenecer a determinado grupo. En el entendimiento de la autora, esta omisión tiene causa en la desconsideración de esos incidentes como una cuestión de injusticia social. La violencia como forma de opresión se caracteriza por la potencialidad y por la predisposición compartida por los miembros del grupo de ser sujetos de violencia. Así, toda mujer sabe que potencialmente puede ser víctima de violación y ese hecho, de poder ser victima, ya la priva de libertad y dignidad. La violencia cuanto a los grupos es una práctica social y cuando realizada en grupos, muchas veces es tolerada. No existe racionalidad en esta violencia. La violencia del Estado, al contrario, aún que perversa se sustenta por motivos particulares del grupo dominante. La violencia como "cara” de la opresión y de la injusticia no se encuentra contemplada en la noción distributiva de la justicia.

Considerando que es en el contexto de la sociedad de bienestar capitalista que se desarrolla con mayor intensidad las discusiones sobre las teorías de la justicia, Young sustenta que "el paradigma distributivo de justicia es la formulación más importante del debate publico mantenido en tales sociedades", bien como "que el paradigma distributivo de la justicia funciona ideológicamente en el sentido de reforzar la despolitización”. La sociedad del bienestar capitalista al revés de la capitalista liberal donde impera el laissez faire tiene tres postulados principales: (a) la actividad económica debe ser regulada en el quantum necesario al desarrollo social y de la obtención del bienestar colectivo; (b) la ciudadanía comprende la satisfacción de las necesidades básicas, para tanto actuando el Estado, se necesario, mediante políticas propias; (c) la observancia del principio de la igualdad formal y de la impersonalidad. La complexidad de la economía capitalista impone algún tipo de reglamentación por el Estado, lo que existe en casi todos los países de la Europa. No es un "socialismo progresivo" pues los objetivos son establecer condiciones benéficas a la acumulación de capitales, todavía, son positivos pues preexiste un cierto controle de la economía por el Estado y los ciudadanos necesitan de buenas condiciones económicas para su sustento y bienestar. En las sociedades capitalistas contemporáneas el gran beneficiario del Estado es la empresa privada. El Estado asume los costos de la formación de los trabajadores, tiene el encargo del sistema de transporte que garante la circulación del producto y aún es el principal consumidor del producto. La sociedad capitalista acepta que la satisfacción de las necesidades básicas de los ciudadanos cuando no hechas por las instituciones privadas, sean hechas por el Estado que actúa a través de políticas como por ejemplo, los planos de asistencia. En la sociedad del bienestar se disfruta de una correlación entre la acumulación de capital y la satisfacción de las necesidades 
de los ciudadanos, ello porque los programas estatales son financiados con los valores que son aportados por la empresa privada con el pagamento de los impuestos. Fundase por ultimo, el Estado del bienestar en la igualdad formal y de procedimiento. Sin embargo, cambia la forma autoritaria y personalizada y crease una burocracia, que en principio, debe tratar todos por igual y la división de poder se opera en razón de la posición del trabajo en la jerarquía burocrática y no en razón de condiciones personales. Según Young, las instituciones y las prácticas del Estado de bienestar ayudan a preservar las instituciones capitalistas. Favorecen la producción y la acumulación, ayudan a proporcionar una fuerza de trabajo especializada y amplían los mercados de bienes a través de ayudas directas al consumo. Con las políticas de ayuda el Estado busca legitimar el sistema mediante la gestión de la lealtad del ciudadano que recibe del Estado beneficios materiales. Es de considerase, también, que la mayor parte de las políticas del Estado de bienestar surgieran de luchas del pueblo contra los ricos y poderosos. Esta actuación del Estado por razón de regulación de la economía y de políticas de satisfacción de necesidades lleva a la despolitización. No se cuestionan las formas de organización y los objetivos de la producción. El conflicto social y las discusiones se limitan a la cuestión distributiva. Hay un ejemplo concreto de pacto en este sentido, como lo informa Young: "En el sector privado, después de la segunda guerra mundial los sindicatos estuvieran implícitamente de acuerdo en restringir sus demandas a cuestiones distributivas - salarios, horas, beneficios, vacaciones - y no sacar a colación cuestiones como las condiciones de trabajo, el control del proceso de producción o las prioridades de inversión (Bowles y Gintis). La regulación estatal de la negociación colectiva ha reforzado este acuerdo implícito, no permitiendo casi que se introdujeran en la agenda de negociación cuestiones relativas a los procedimientos y la organización laboral”. Restrictos en la sociedad del bienestar los conflictos y las decisiones políticas a la cuestión productiva, los ciudadanos son clientes - consumidores. El propio ciudadano en razón de los reclamos del consumo se percibe como un consumidor que se agrupa, se organiza para así recibir beneficios del Estado. En la sociedad capitalista del bienestar el vehículo para la solución de los conflictos políticos es el grupo. El pluralismo se expresa a través del interés del grupo lo que es contestado por algunos que entienden que el sistema provoca la injusticia distributiva. Young acepta esta tesis, entendiendo y acrecentando que el pluralismo fundamentado en el interés del grupo lleva a la despolitización de la vida publica. No hay justicia en el pluralismo de los intereses del grupo. La participación, por grupo, en la discusión de los conflictos no permite la participación de la persona individualmente considerada, lo que justifica la poca participación de las mujeres condición que lleva a la despolitización, ahí, Ágnes Heller va a afirmar, "que el predominio de un paradigma distributivo entre las teorías de justicia de la filosofía contemporánea puede explicarse, al menos en parte, por el hecho de que en la sociedad capitalista de bienestar las cuestiones distributivas dominan la discusión política". 8 La sociedad capitalista crea nuevas formas de opresión. La burocracia, que fue idealizada como una forma de eliminar la dominación, principalmente la de clase, pero se constituye en una nueva forma de

${ }^{8}$ Cf., a respecto el importantísimo artículo de Ágnes Heller, Sociología de la vida cotidiana. Barcelona: Ed. Península, 1998. 
opresión en la medida que hace con las personas estén sometidas a la disciplina de las autoridades. La burocracia bajo el imperio de la ley y de los procedimientos retoma la soberanía personal propia de las formas tradicionales de dominio. Las normas son de carácter general y deben ser observadas por cualquiera que ejerce el puesto o desarrolla la tarea introduciendo la generalización de la actividad social o cooperativa. La dominación originada por la burocracia no se limita al trabajo, ocurre también en otros aspectos de la vida como sumisión a la autoridad de hospitales, escuelas, establecimientos financieros etc. La dominación, todavía, no es cuestionada y ello puede acreditar quien sabe al precio a ser pago por lo conforto.

En los años 60 y 70 el movimiento contracultural hizo del cuerpo y sus adornos motivo de lucha. Los hippies alteraran los padrones de la vestimenta, los punks continuaran el movimiento creando todo un estilo de vida. Hasta los hábitos alimentares fueran alterados con la llamada alimentación natural. El feminismo movimiento más que cultural es indubitablemente el más significativo. Surgieran también, movimientos de minorías raciales y étnicas oprimidas, de mayores y otros que politizaran la cultura pues cuestionaran los estereotipos. Los movimientos surgidos en final de los años 60 romperán los parámetros anteriores y pasaran por cuestionar el sistema. Fueran allá de las cuestiones distributivas discutiendo la organización del poder en las instituciones. La sociedad reaccionó algunas veces con violencia, otras integrando las pretensiones. Releva notar que la democracia es elemento de la justicia pues reduce la dominación. Por eso tiene valor no solo intrínseco como también instrumental. La participación es un instrumento que asegura a los ciudadanos exponer y buscar atendimiento de sus necesidades. El valor intrínseco está en la existencia de la posibilidad de participar. El simple saber sobre la posibilidad de participar lleva a pensar sobre las propias necesidades. La democracia implica participación lo que, sin embargo, no asegura la ausencia de opresión. La decisión tomada de forma democrática mediante la participación efectiva del ciudadano puede resultar en ley y procedimiento que genere opresión para determinado grupo. Así, no se debe dejar de considerar las objeciones que son hechas cuanto a la afirmativa de que procedimientos democráticos en el ámbito de la tomada de decisión asegure la justicia. Para que se pueda alcanzar la justicia por vía de la democratización de las decisiones es necesario que la democracia sea constitucional, que las reglas estén previamente decididas con derechos básicos asegurados. Por ultimo, es necesario que la democracia alcance no-solamente las instituciones políticas como también, las corporaciones privadas y la burocracia. De manera reflexiva afirma Young, con razón, que solo en un ámbito publico heterogéneo que reconozca las diferencias es posible hablar de justicia.

Reflejando sobre la obra de Young, recordemos Antonio G. Robledo, en su libro, Meditación sobre la Justicia, ${ }^{9}$ que con propiedad expone: "En la filosofía helenística primero, y en la patrística después, Aristóteles pasó siempre, al igual que Platón, por haber hecho de la justicia uno de los objetos predilectos de su especulación. 'La mayoría de los filósofos - dice Lactancio - pero sobre todo Platón y Aristóteles, han hablado abundantemente de la justicia; han sido ellos los defensores de esta

\footnotetext{
${ }^{9}$ México: Fondo de Cultura Económica, 1963, p. 41
} 
virtud a la que colmaron de elogios insignes al decir que es ella la que da a cada cual lo suyo y que mantiene en todas las cosas la igualdad.' Lo primero: suum cuique, es, la vieja fórmula de Simónedes grandiosamente elaborada por Platón. Lo segundo, en cambio: quod aequitatem in omnibus servet (donde aequitas es sinónimo de aequalitas), es la aportación original de Aristóteles, para el cual, y con las presiones que luego diremos, la justicia consiste ante todo en la igualdad”. Igualdad, respetadas las diferencias. Para hablar de justicia es preciso hablar de derecho, hablar de norma jurídica que fundamenta la pretensión de alguno. La eficacia de las normas jurídicas supone la tendencia, consustancial de todo sistema jurídico, a realizarse socialmente de una manera efectiva, así, "la faceta de eficacia que acompañada las de validez y de justicia, tantas veces se ha traído a colación entre los filósofos del derecho, expresa no sólo un síntoma de la existencia de una norma jurídica, sino una pretensión inherente a la propia aparición de la norma", ${ }^{10}$ lo que da a nosotros la certeza que derecho sin justicia no es derecho y justicia sin reconocimiento de las desigualdades, es la más pura forma de arbitrio.

En el relato de Iris Marion Young, podemos concluir que Justicia social, democracia y los derechos humanos son conceptos intercambiables. No cabe la realización de unos sin los otros. El concepto de derechos humanos implica democracia y estado de derecho, y a su vez los derechos humanos sólo son garantizados en un régimen democrático, en una democracia que aspira y posibilita continuamente a mayores cotas de participación del ciudadano en la economía, la cultura y la política. Los extraordinarios y continuos avances científico-tecnológicos están modificando las condiciones de vida de las personas, su forma de trabajar, consumir, divertirse, comunicarse, etc., y a la vez plantean nuevos riesgos y exigencias. Por poner un ejemplo, los conocimientos y tecnologías disponibles en el campo de la genética y biología - genoma humano, ingeniería genética, clonación, trasplantes neuronalessuscitan cuestiones bioéticas fundamentales. La cantidad e intensidad de informaciones que cotidianamente bombardean nuestras mentes con unos medios tan sofisticados y generalizados obstaculizan gravemente el conocimiento sistemático de la realidad, la elaboración de criterios personales, el pensamiento crítico y la toma de decisiones racionales. En la sociedad de la información, la persona reclama el derecho a la verdad de las informaciones, a no ser engañada ni manipulada, a no ser perturbada con propaganda deformante. Reclama el derecho a poder expresar las propias opiniones en unos medios cada vez más cerrados y monopolizados, bien como el derecho a la propia intimidad y a la protección de los datos personales. Ante la globalización de la economía y la política, y en la medida en que el poder económico es cada vez más determinante en las decisiones políticas, el derecho a la participación democrática se extiende más allá del poder político y exige participación en el poder económico. Así como las demandas de los derechos civiles y políticos se concretaron en la revolución liberal-burguesa, y las demandas de protección social en la revolución industrial, la revolución científico-tecnológica están planteando en la actualidad exigencias muy concretas, y en el futuro demandará otras que hoy no se pueden siquiera prever.

${ }^{10}$ Romero Moreno, J.M. Proceso y Derechos Fundamentales en la España del siglo XIX. Madrid: Centro de Estudios Constitucionales, 1983, p. 18. 